Check for updates

Cite this: RSC Adv., 2019, 9, 4765

Received 21st December 2018

Accepted 30th January 2019

DOI: 10.1039/c8ra10490e

rsc.li/rsc-advances

\section{Preparation and characterization of POSS- containing poly(perfluoropolyether)methacrylate hybrid copolymer and its superhydrophobic coating performance}

\author{
Zhujun Lyu, (D) * Qiufeng An,* Pengwei Qin, Weiwei Li and Xiaoge Wang
}

To design a mechanically stable and superhydrophobic coating, a polyhedral oligomeric silsesquioxane (POSS)-containing poly(perfluoropolyether)methacrylate (PFPEM) hybrid copolymer (PFPEM-POSS) was synthesized via a free-radical solution polymerization with PFPEM, $1 \mathrm{H}, 1 \mathrm{H}, 2 \mathrm{H}, 2 \mathrm{H}$-perfluorooctyl acrylate, methyl (meth)acrylate, $n$-butyl acrylate, hydroxypropyl acrylate, methacryloxy propyl trimethoxy silane, and methacrylisobutyl POSS; and azobisisobutyronitrile as an initiator. Hydrophobic coatings were formed on substrates by a facile one-step dip-coating method in a solution mixture of diethylene glycol dimethyl ether with the PFPEM-POSS hybrid copolymer. The chemical structure of the PFPEM-POSS copolymer and the surface morphology and performance of the PFPEM-POSS coatings were investigated. The results indicate that, under POSS aggregation via the fluorophilic/oleophilic comonomer phase separation and owing to the low-surface-energy poly(perfluoropolyether)methacrylate incorporated into the copolymer, PFPEM-POSS exhibited a hierarchical micro-nano roughness in atomic force microscopy observations and provided the treated substrates with excellent hydrophobicity and abrasion resistance. As a result, the water contact angle reached $152.3^{\circ}$ on the treated glass.

\section{Introduction}

Inspired by self-cleaning and anti-fouling properties observed in nature (i.e., lotus leaves and water striders), tremendous effort has been made during the past several decades to fabricate biomimetic superhydrophobic surfaces. ${ }^{\mathbf{1 - 4}}$ The wetting behavior of surfaces is dominated by surface chemistry and roughness, ${ }^{5}$ and it is well-known that a low-energy surface can reduce intermolecular attractive forces. ${ }^{6}$ Furthermore, in materials with contact angles (CAs), for untreated substrates, greater than $90^{\circ}$, an increased surface roughness leads to a decrease in surface energy that results in a larger static CA. ${ }^{7}$ Therefore, incorporating nanoparticles to provide surface roughness combined with low-surface-energy fluorinated surfaces or organosilicon compounds via physical blending or chemical bonding can build hydrophobic coatings. ${ }^{8}$

Generally, increasing roughness is a prerequisite for achieving superhydrophobicity, but it also induces a reduction of the transmittance owing to light scattering. ${ }^{9}$ Moreover, surfaces with large roughness normally exhibit poor mechanical durability. ${ }^{\mathbf{1 0 - 1 2}}$ Reduced roughness increases the contact area between liquids and surfaces, whereby the hydrophobicity would be easily lost. ${ }^{13}$

Key Laboratory of Auxiliary Chemistry \& Technology for Chemical Industry, Ministry of Education, Shaanxi University of Science and Technology, Xi'an, Shaanxi, 710021, China. E-mail: anqf@sust.edu.cn; Tel: +86-1580-922-5566
Hierarchical micro-nano scale morphology structures are ideal for resisting contamination or abrasion damage, ${ }^{\mathbf{1 4}, 15}$ and are more likely to maintain surface roughness after damage than single structures. ${ }^{\mathbf{1 6}}$ Among hybrid organic-inorganic building blocks, polyhedral oligomeric silsesquioxane (POSS) has attracted significant research effort. This is owing to the feature of POSS compounds to easily link covalently into a polymer backbone, ${ }^{17}$ and the result wherein the incorporation of POSS as nanoparticles into polymers induces good mechanical strength and thermal and chemical resistance. ${ }^{18}$

A number of reports have described the preparation of fluorinated POSS coatings. For example, Iacono et al. prepared superhydrophobic coatings by solvent blending fluorinated POSS with perfluorocyclobutyl aryl ether polymers. ${ }^{19}$ Further, Wang et al. successfully formed self-healing superhydrophobic and superoleophobic coatings by introducing heptadecafluorodecyltethered polyhedral oligomeric silsesquioxane (FD-POSS). ${ }^{\mathbf{2 0}}$ Ganesh et al. coated fluorinated polyhedral oligomeric silsesquioxanes-poly(vinylidene fluoride-co-hexafluoro propylene) via electrospinning to achieve transparent superhydrophobicity. ${ }^{21}$ Finally, Skrzypiec et al. published a study describing the production of highly hydro/oleo-phobic and self-cleaning coated surfaces based on the incorporation of heptadecafluorodecyltethered polyhedral oligomeric silsesquioxane (FD-POSS) into the perfluorocyclopentenyl (PFCP) aryl ether polymer. ${ }^{22}$ However, few reports exist regarding research on superhydrophobic 
coatings comprising a POSS-containing poly(perfluoropolyether) methacrylate (PFPEM) copolymer (PFPEM-POSS). In particular, PFPEM exhibits very low surface energies $\left(12-20 \mathrm{mN} \mathrm{m}^{-1}\right)$, and a high chemical stability via the strong $\mathrm{C}-\mathrm{C}$ and $\mathrm{C}-\mathrm{F}$ bonds. Additionally, PFPEM presents interesting characteristics such as biocompatibility, biological inertness, and the lack of potential teratogenic and carcinogenic harm to organisms. ${ }^{23-26}$ Therefore, to obtain superhydrophobicity and durability in coating fabrication, POSS incorporated into the PFPEM copolymer via freeradical solution polymerization was designed and synthesized. On the basis of this, a superhydrophobic coating with a high adhesion to glass substrates was prepared.

\section{Experimental}

\section{Materials and reagents}

The poly(perfluoropolyether)methacrylate (PFPEM) was purchased from Hunan Nonferrous Chenzhou Fluorine Chemistry Co., Hunan, China. $1 H, 1 H, 2 H, 2 H$-Perfluorooctyl acrylate (C6F) was supplied by Fuxin Hengtong Chemical Co., Fuxin, China. Methacrylisobutyl POSS (MAPOSS) was purchased from Hybrid Plastics, US. 3-Methacryloxypropyltrimethoxysilane (KH570) was obtained from Nanjing Yudeheng Co., Nanjing, China. Hydroxypropyl acrylate (HPA) was supplied by Tianjin 6th Chemical Factory, Tianjin, China. 2,2'-Azobis(isobutyronitrile) (AIBN) was purchased from Shijitongda Chemical Co., Jinan, China, and was recrystallized thrice from anhydrous ethanol. Hydrofluo-roether (7200) was obtained from 3M Novec $^{\mathrm{TM}}$, US. HDI trimer Desmodur N3390 was supplied by Bayer Co., Germany. Methyl (meth)acrylate (MMA), n-butyl acrylate (BAc), acetone and diethylene glycol dimethyl ether (DGME) were provided by Tianjin Kemiou Chemical Reagent Co., Tianjin, China. All reagents were used as received.

\section{Synthesis of poly(MMA-co-BAc-co-HPA-co-KH570-co-PFPEM- co-C6F-co-MAPOSS)}

In a three-neck flask equipped with a thermometer, a mechanical stirrer, and a reflux condenser, $7.37 \mathrm{~g} 7200,8.08 \mathrm{~g}$ C6F and $6.67 \mathrm{~g}$ BAc were mixed together as a solvent. Further, $3.00 \mathrm{~g}$ MAPOSS, $3.06 \mathrm{~g}$ MMA, $2.11 \mathrm{~g}$ KH570, $1.03 \mathrm{~g}$ HPA, and 2.02 g PFPEM were employed as co-monomers. The initiator, AIBN, accounted for $1 \mathrm{wt} \%$ of the co-monomers. The general procedure was as follows: first, the flask was filled with $30 \%$ mixed solvent and, after being stirred for $10 \mathrm{~min}$, the mixture was then heated to $70{ }^{\circ} \mathrm{C}$ under nitrogen protection. Second, the AIBN was added into the flask. In a clean beaker, all of the monomers were dissolved into $70 \%$ solvent and, after fully mixed, added in dropwise fashion to the flask during a duration of 4-5 h. After the addition of all materials, the reaction was maintained for $4 \mathrm{~h}$ at $70{ }^{\circ} \mathrm{C}$. Ultimately, a transparent to slightly yellow poly(MMA-co-BAc-co-HPA-co-KH570-coPFPEM-co-C6F-co-MAPOSS) solution was obtained and designated as PFPEM-POSS (Scheme 1).

\section{Preparation of the PFPEM-POSS coatings}

Pre-treatment of the glass. Prior to the coating treatment, the glass pieces were washed with detergent and deionized water,

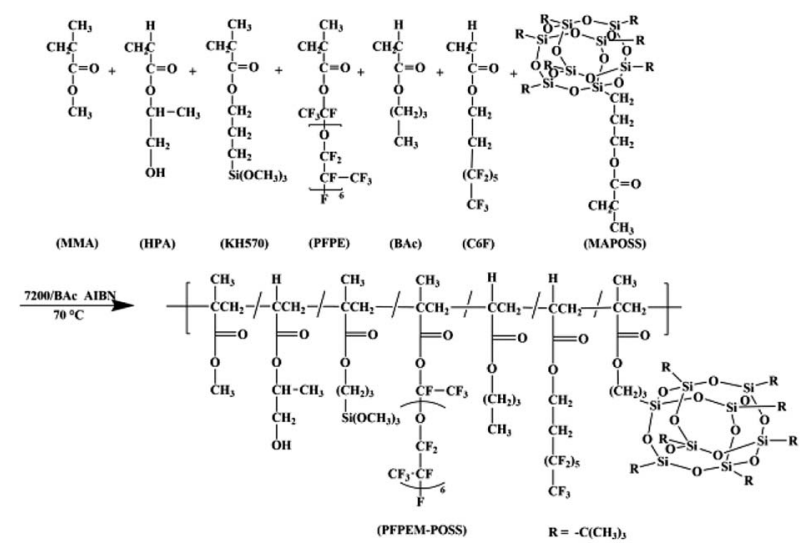

Scheme 1 Schematic illustration of the PFPEM-POSS synthesis procedure.

and then ultrasonically cleaned in acetone for $30 \mathrm{~min}$ to remove any oil residue and dirt. After being dried at $100-105{ }^{\circ} \mathrm{C}$ for $20 \mathrm{~min}$, the cleaned glass was etched by oxygen plasma in air atmosphere for $20-30 \mathrm{~s}$ at a gas flow rate of about $1.69 \times 10^{-1} \mathrm{~Pa}$ $\mathrm{m}^{3} \mathrm{~s}^{-1}$.

Coating preparation. A flow chart outlining the preparation of the superhydrophobic coating is given in Scheme 2. First, the PFPEM-POSS was dissolved and diluted with DGME to form a treatment solution with a mass concentration of $0.8 \%$. Next, the curing agent N3390 was added and fully stirred. The $n(\mathrm{OH})$ in PFPEM-POSS $/ n$ (NCO) in the N3390 was about 1.1 : 1.0. Subsequently, the obtained treatment solution was dispersed for $30 \mathrm{~min}$ in an ultrasonic bath, whereupon the etched glass was dipped into the treatment solution to form a coating. The duration of the initial dip was $3 \mathrm{~min}$, followed by a dip $10 \mathrm{~s}$ in duration that was repeated 3-4 times. The thickness of the coatings was 10 $\mu \mathrm{m}$. After being held at room atmosphere for $3 \mathrm{~min}$, the coated glass was placed into an oven and cured at $150{ }^{\circ} \mathrm{C}$ for $30 \mathrm{~min}$. Finally, the PFPEM-POSS coating anchored on the glass substrate was stained and held in a desiccator until it was used.

\section{Characterization}

The Fourier transform infrared (FT-IR) spectra of the samples were recorded between 4000 and $400 \mathrm{~cm}^{-1}$ on a VERTE-70

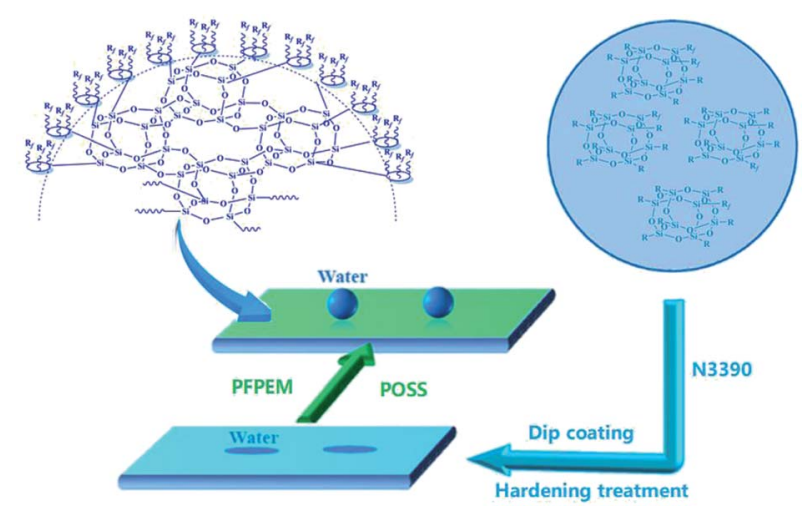

Scheme 2 Schematic illustration of the superhydrophobic coating fabrication process. 
spectrophotometer (Bruker, Germany) using the $\mathrm{KBr}$ pellet technique. The proton nuclear magnetic resonance $\left({ }^{1} \mathrm{H}-\mathrm{NMR}\right)$ analysis was performed with an INOVA-400 spectrometer (Bruker, Germany) using $\mathrm{CDCl}_{3}$ as a solvent and tetramethylsilane as an internal standard. The scanning electron microscope (SEM) measurements were carried out on a TM-1000 SEM (Hitachi, Tokyo, Japan), and the morphology images were obtained at a magnification of $1000 \times$. Atomic force microscopy (AFM) images were obtained with a Nanoscope IIIA AFM (Agilent Technologies, Palo Alto, CA, USA) in tapping mode. All scanning was performed at $22{ }^{\circ} \mathrm{C}$ in air with a relative humidity of $48 \%$. The chemical structure of the PFPEM-POSS film on the treated glass was investigated by an Axis Ultra X-ray photoelectron spectrometer (XPS) made in Kratos, Manchester, UK. The static water CAs (WCAs) on the glass treated with PFPEM-POSS were determined via the sessile drop method on a JC2000A CA goniometer (Shanghai Zhong Chen Digital Co., Shanghai, China) at $20^{\circ} \mathrm{C}$. The liquid volume was $5 \mu \mathrm{L}$, and an average of five readings from different regions of the same sample was used as the final CA value for each sample. Spectral transmittances of wavelengths between 300 and $800 \mathrm{~nm}$ were measured using a Cary 5000 ultraviolet-visible spectrophotometer (Agilent Co., Palo Alto, CA, USA), with blank glass as a control. Abrasion resistance analysis was done using an LKY-II wet abrasion scrub tester (Hongwei Co., Dongguan, Guangdong, China) with a $500 \mathrm{~g}$ weight and cotton cloth used as the abrasion material.

\section{Results and discussion}

\section{Design and synthesis of PFPEM-POSS}

Various practical and synthetic considerations may hold significant sway over the surface composition, wettability, and adhesion characteristics of polymeric coatings such as those studied herein. ${ }^{27}$ To determine the ideal chemical and mechanical properties for superhydrophobic coatings, several monomers were incorporated to combine various properties and thus optimize the PFPEM surface enrichment. The basic design was a random polymer with three variable monomer classes. The first monomer class were oleophilic alkyl(meth)acrylates; specifically, MMA and BAc. This monomer class was chosen for the good mechanical strength and biocompatibility at room temperature exhibited by its homopolymers. ${ }^{28}$ In addition, MAPOSS was used herein, which not only displayed good mechanical strength akin to MMA, it also led to cage-like nanocomposites that enhanced the coating roughness. MAPOSS as hybrid material is a highly crosslinked network macromolecule, which is easy to cure. The second monomer class was the fluorophilic PFPEM macromonomer and C6F. The incorporation of this monomer class provided for a highlysurface-active, low-energy, fluorinated component. The low surface tensions associated with fluoropolymers provided a driving force for the observed surface enrichment of the PFPEM domains in these studies. ${ }^{29}$ In addition, polymer surfaces rich in fluorinated functional groups can be envisioned to express other properties upon the solid surface, such as chemical resistance, hydrophobicity, and a low coefficient of friction. The combination of the fluorophilic and oleophilic components led to micro-phase separating morphologies. The less hydrophobic POSS was mostly buried below the film surface. Finally, MAPOSS aggregation reinforced the copolymer and thus contributed to the abrasion resistance of the coating. Furthermore, the coating could be characterized by hierarchical micro-nano roughness that induced positive effects on both the static CA and mechanical durability of the coatings. The third monomer class was reactive monomers that contained curable functional groups. Using various compositions and curing conditions, the relative effect of cross-link density was evaluated. The HPA component could provide a crosslinkable functional group $(-\mathrm{OH})$ and facilitate copolymer crosslinking with a triisocyanate curing agent. The trialkoxysilyl groups from KH570 could bond to glass and assure a good coating adhesion. In addition, BAc and C6F could be used both as co-monomers and mixed as cosolvents with 7200 to assist in the complete dissolution of PFPEM with the other co-monomers.

\section{Characterization of PFPEM-POSS}

Fig. 1 and 2 show the FT-IR and ${ }^{1} \mathrm{H}-\mathrm{NMR}$ spectra of the PFPEMPOSS, respectively. The FT-IR spectra of the PFPEM-POSS (Fig. 1a) and the monomer MAPOSS (Fig. 1b) all exhibit the characteristic stretching and distortion vibration peaks assigned to $\mathrm{Si}-\mathrm{O}, \mathrm{Si}-\mathrm{C}, \mathrm{C}=\mathrm{O}$ and $\mathrm{C}-\mathrm{H}$ bonding. These result from the skeleton and side groups of MAPOSS, and occurred at $1061\left(V_{\mathrm{Si}-\mathrm{O}}\right), 1290\left(V_{\mathrm{Si}-\mathrm{C}}\right), 1731\left(V_{\mathrm{C}-\mathrm{O}, \mathrm{C}=\mathrm{O}}\right), 2943-2845\left(V_{\mathrm{C}-\mathrm{H}}\right.$, $\left.-\mathrm{CH}_{3} /-\mathrm{CH}_{2}\right)$ and $1453-1401\left(\delta_{\mathrm{C}-\mathrm{H}},-\mathrm{CH}_{3}\right) \mathrm{cm}^{-1}$. In the PFPEMPOSS spectrum, the adsorption at $3463 \mathrm{~cm}^{-1}$ can be attributed to the $-\mathrm{OH}$ groups of HPA. The stretching vibration of $\mathrm{C}-\mathrm{F}$ and the symmetric and asymmetric stretching vibrations of $\mathrm{C}-\mathrm{O}$ were overlapped resulting in broadening of the absorption peaks at $1244-1122 \mathrm{~cm}^{-1} .^{30}$ The strong signal at $809 \mathrm{~cm}^{-1}$ and the weak absorption band at 1244 and $1192 \mathrm{~cm}^{-1}$, however, were assigned to the $-\mathrm{CF},-\mathrm{CF}_{3}$, and $-\mathrm{CF}_{2}$ groups from PFPEM and $\mathrm{C6F}$. Moreover, the peak attributed to $\mathrm{C}=\mathrm{C}\left(1636 \mathrm{~cm}^{-1}\right)$ appeared in the MAPOSS spectrum but was almost nonexistent in the PFPEM-POSS spectrum, verifying that the PFPEM, C6F, MMA, BAc, HPA, KH570 and MAPOSS have been well copolymerized.

Fig. 2 shows the ${ }^{1} \mathrm{H}-\mathrm{NMR}$ spectrum of PFPEM-POSS, where the ${ }^{1} \mathrm{H}-\mathrm{NMR}$ (ppm) $\left(300 \mathrm{MHz}, \mathrm{CDCl}_{3}\right)$ of PFPEM-POSS exhibited

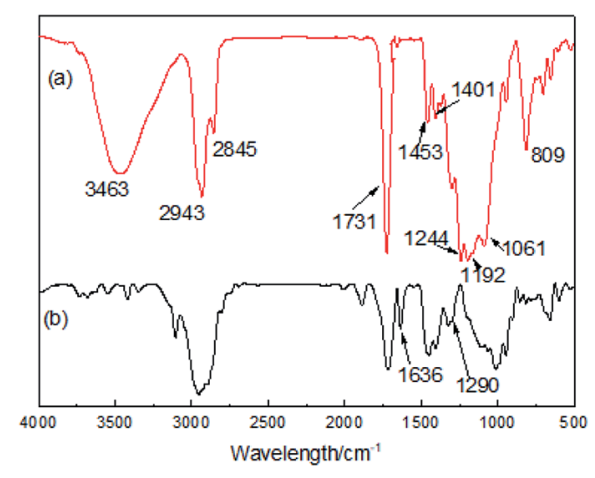

Fig. 1 FT-IR spectra of (a) PFPEM-POSS and, (b) MAPOSS. 

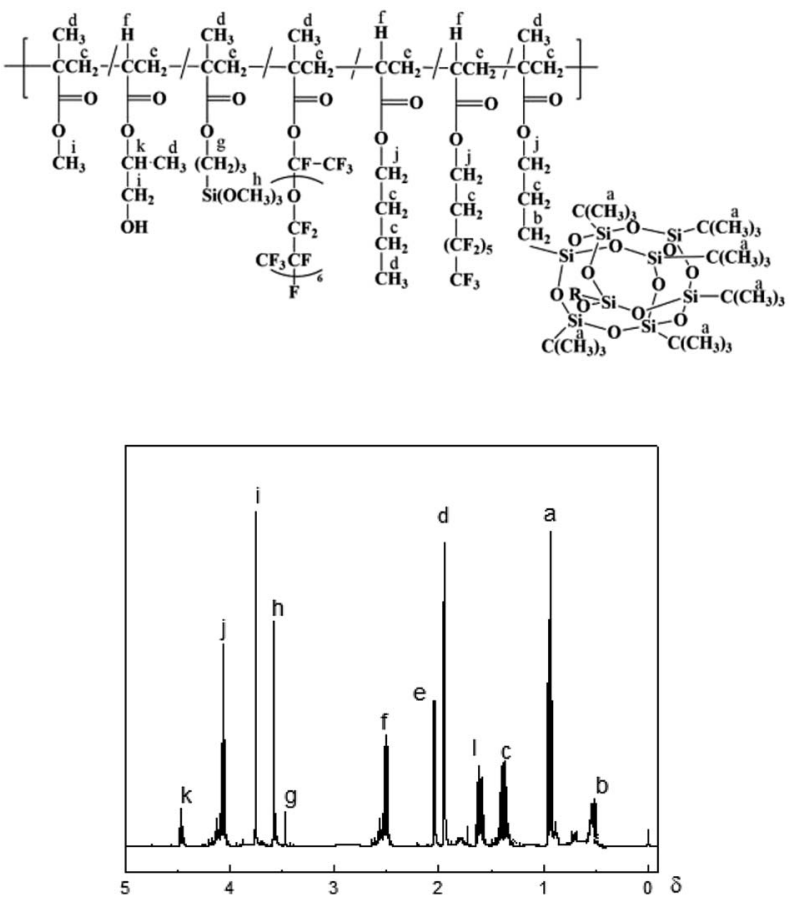

Fig. $2{ }^{1} \mathrm{H}-\mathrm{NMR}$ spectrum of PFPEM-POSS.

a chemical shift, $\delta$, at $4.06\left(\mathrm{jH},-\mathrm{C}_{2} \mathrm{O}-\right), 1.37\left(\mathrm{cH},-\mathrm{CH}_{2} \mathrm{C}_{2}{ }^{-}\right.$ $\left.\mathrm{CH}_{2}-\right), 0.94\left(\mathrm{aH},-\mathrm{C}\left(\mathrm{CH}_{3}\right)_{3}\right), 1.60\left(\mathrm{cH}, \mathrm{Si}-\mathrm{CH}_{2} \mathrm{CH}_{2}\right)$, and $0.54(\mathrm{bh}$, $\left.\mathrm{Si}-\mathrm{CH}_{2} \mathrm{CH}_{2}\right)$. The shift peak $\delta 3.57(\mathrm{hH})$ belonged to the $\delta_{\mathrm{H}}$ of $-\mathrm{OC} \underline{\boldsymbol{H}}_{3}$ from $\mathrm{KH} 570$, while that at $\delta 4.49(\mathrm{kH})$ was assigned to the $\delta_{\mathrm{H}}$ from HPA. Finally, the observed shift peak at $\delta 2.52(\mathrm{fH})$ was linked to carbon atoms in $\mathrm{C} 6 \mathrm{~F}, \mathrm{BAc}$ and HPA. In conclusion, both the FT-IR and ${ }^{1} \mathrm{H}$-NMR spectra indicate that the monomers were polymerized together through free-radical solution polymerization.

\section{Morphology and hydrophobic properties of the PFPEM-POSS coating on substrates}

The SEM and AFM images provide two effective methods for observing the morphology of a coating on the micrometer-tonanometer scale. ${ }^{31}$ The SEM image of the non-POSScontaining coating (Fig. 3a) indicates a coating surface that is relatively smooth. The SEM image of the POSS-containing coating (Fig. 3b), however, clearly shows a coating surface with numerous high-contrast micro/nano-scale particle aggregations. Of these structures, the nano-scale protrusions were

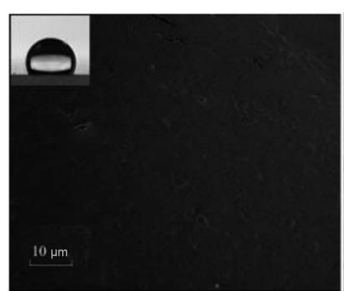

(a)

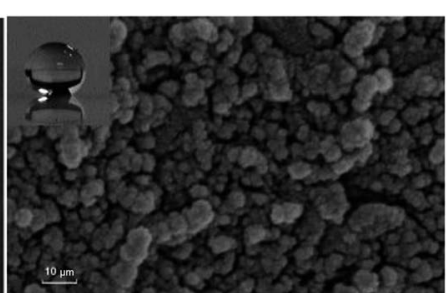

(b)
Fig. 3 SEM images of a silicon wafer coated with (a) poly(MMA-COBAC-CO-HPA-CO-KH570-CO-PFPEM-CO-C6F), WCA = 109.8 ; and with (b) PFPEM-POSS, WCA $=152.3^{\circ}$. caused by POSS agglomeration and the micro-particles resulted from fluorophilic and oleophilic component phases separating the morphologies. It is apparent in Fig. $3 \mathrm{~b}$ that the POSS particle aggregations have changed from a random distribution to superpositional vertical growth, which is similar to the mastoid structure of lotus leaves. Meanwhile, the WCA on this POSScontaining surface could reach $152.3^{\circ}$.

The AFM images in Fig. 4a and $\mathrm{b}$ reveal that the PFPEMPOSS formed a rugged and uneven coating on the silicon wafer substrate where according to SEM analysis, these bulges were caused by POSS aggregation. With a topographical data scale in the $z$-direction of $200 \mathrm{~nm}$ and a scanning field of $2 \times 2 \mu \mathrm{m}^{2}$, numerous nanoparticles were detected in the PFPEM-POSS coating. The root mean square roughness of the PFPEM-POSS reached about $15.2 \mathrm{~nm}$, demonstrating that the nanoscale roughness was successfully combined with the microscale roughness on the coating surface. Such hierarchical micro-nano roughness greatly increased the superhydrophobicity of the fabricated coating.

Fig. 5 gives the wide-scan and high-resolution C1s XPS spectra of PFPEM-POSS. Four strong characteristic peaks were apparent in the wide-scan XPS (Fig. 5a) that were attributed to fluorine, oxygen, carbon, and silicon. These peaks occurred at binding energies of about 687.00 (F1s), 530.00 (O1s), 283.00 (C1s), and 100.00 (Si2p) eV, respectively. Based on the individual peak areas and the corresponding atomic sensitivity factors, the atomic concentrations of $\mathrm{C}, \mathrm{O}, \mathrm{F}$ and $\mathrm{Si}$ in the outermost surface comprising PFPEM-POSS are 47.69, 20.41, 25.4 and 6.5\%, respectively. It is clear in Fig. $5 \mathrm{~b}$ that the C1s spectra can be deconvoluted into seven peaks located at binding energies of about 283.60, 284.80, 286.20, 288.73, 291.27, 293.22 and $293.89 \mathrm{eV}$. These peaks were attributable to the bonds of $\mathrm{C}-\mathrm{Si}$, $\mathrm{C}-\mathrm{C} / \mathrm{C}-\mathrm{H}, \mathrm{C}-\mathrm{OH} / \mathrm{C}-\mathrm{O}, \mathrm{O}-\mathrm{C}=\mathrm{O}, \mathrm{C}-\mathrm{F}(\mathrm{CF}), \mathrm{C}-\mathrm{F}\left(\mathrm{CF}_{2}\right)$, and $\mathrm{C}-\mathrm{F}$ $\left(\mathrm{CF}_{3}\right)$, respectively, and consistent with those reported in

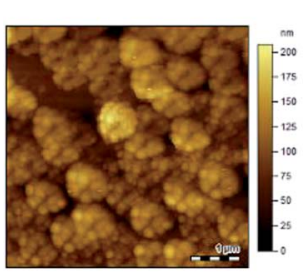

(a)

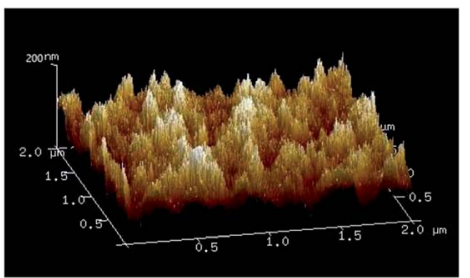

(b)
Fig. 4 AFM topographic images of the PFPEM-POSS coating on a silicon wafer: (a) two-dimensional; (b) three-dimensional.

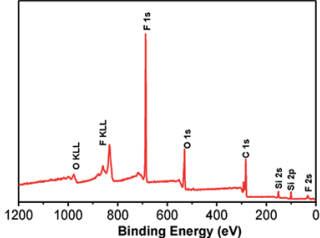

(a)

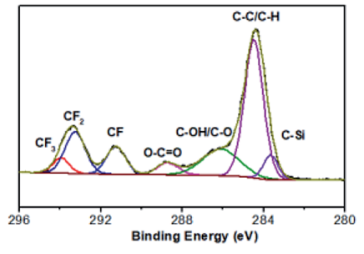

(b)
Fig. 5 XPS spectra of PFPEM-POSS on the silicon wafer: (a) wide-scan spectrum; (b) high-resolution C1s spectrum. 


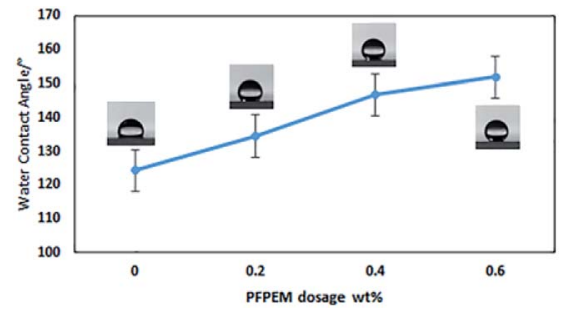

Fig. 6 Water contact angle of the coated surface as a function of the PFPEM dosage in the coating. (Insets) photographs of water droplets on each surface.

literature..$^{32}$ The above results clearly indicate a high atomic ratio of fluorine on the surface owing to the significant surface enrichment of low-surface-energy PFPEM and C6F.

\section{Coating hydrophobicity with varying PFPEM dosage}

It has previously been reported that the trifluoromethyl groupterminated surface possesses the low-surface-energy. However, superhydrophobicity can be achieved through a complementary combination of the $-\mathrm{CF}_{3}$ terminal functionality and hierarchical surface roughness. ${ }^{33}$ In this research, the $-\mathrm{CF}_{3}$ was obtained mainly from PFPEM macromonomer. Therefore, Fig. 6 shows the WCAs of glass surfaces coated with the PFPEM-POSS with varying amounts of PFPEM. It is seen that, as the PFPEM dosage increased, the hydrophobicity of the coated glass surface increased. When the PFPEM dosage was increased to $0.6 \mathrm{wt} \%$, the treatment solution appeared transparent and homogenous and the WCA of the resultant coating reached $152.3^{\circ}$. However, as the dosage was increased above $0.6 \mathrm{wt} \%$, the excessive amount of PFPEM was difficult to dissolve with the other comonomers because of its significantly higher molecular weight. Therefore, the excess PFPEM sank to the bottom of the flask and the treatment solution became feculent, which produced an opaque coating with visible crystalline aggregates on the surface. Therefore, $0.6 \mathrm{wt} \%$ PFPEM was adopted herein to fabricate the superhydrophobic coating.

\section{Performance of the PFPEM-POSS coating}

The PFPEM-POSS treatment solution was prepared from the POSS-containing poly(perfluoropolyether)methacrylate hybrid copolymer and HDI trimer N3390 curing agent. The mass concentration of the treatment solutions with diethylene glycol dimethyl ether solvent was $0.8 \%(\mathrm{w} / \mathrm{w})$. The temperature and curing time of the coating was $150{ }^{\circ} \mathrm{C}$ and $30 \mathrm{~min}$, respectively, and the coating method used was dipping. The result is shown in Table 1.

Table 1 Performance of the PFPEM-POSS coating ${ }^{a}$

\begin{tabular}{llllll}
\hline$w \%$ & WCA & OCA & $T \%$ & Appearance & $\begin{array}{l}\text { Abrasion } \\
\text { resistant/cycles }\end{array}$ \\
\hline
\end{tabular}

$\begin{array}{lllllll}\text { PFPEM-POSS } & 0.8 & 152.3 & 87.5 & 95 & \text { Transparent } & 5000\end{array}$

${ }^{a} w \%$ : mass of PFPEM-POSS per $100 \mathrm{~g}$ diethylene glycol dimethyl ether solvent; WCA: water contact angle; OCA: oil contact angle (sunflower oil); $T$ : transmittance.

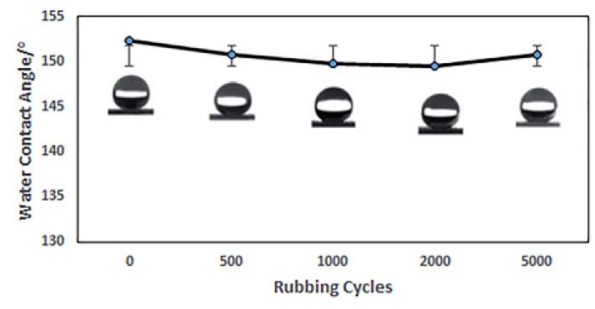

Fig. 7 Water contact angle of the coated surface as a function of the number of abrasion test cycles. (Insets) photographs of water droplets on each surface.

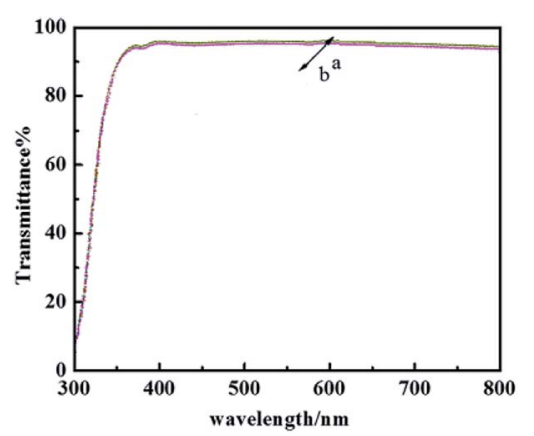

Fig. 8 Transmittance of the coatings: (a) blank, and (b) PFFEM-POSS.

The hydrophobicity of the coating was assessed with WCA measurements, and the data summarized in Table 1 show that the glass treated with PFPEM-POSS has possessed robust superhydrophobicity. Fig. 7 shows the WCA values of the PFPEM-POSS-coated surface during 5000 abrasion test cycles, where the WCA is seen to change only slightly and decrease by $0.99 \%$ after 5000 cycles. These abrasion tests simulated actual damage and, although the WCA reduced with additional abrasion cycles, the treated glass surfaces can withstand at least 5000 cycles of abrasion damage without losing their hydrophobicity. Comparing these results with those of the Dow Corning® 2604 Coating, ${ }^{34}$ tested via the same test method, after 5000 abrasion cycles the Dow Corning new anti-fingerprint coating, the WCA decreased by $0.88 \%$. This indicates that the abrasion durability of the PFPEM-POSS coating is practically the same as the Dow Corning ${ }^{\circledR} 2604$ Coating durability. Therefore, the presence of POSS as nanoparticles combined with PFPEM can endow glass surfaces with a highly durable coating. Furthermore, from Fig. 8 demonstrates that the PFPEM-POSS coating exhibits good transmittance $(>90 \%)$ that is nearly equivalent to that of the uncoated glass substrate.

\section{Conclusions}

Herein, a novel POSS-containing poly(perfluoropolyether) methacrylate hybrid copolymer (PFPEM-POSS) was produced using a facile one-step dip-coating method. The polyhedral compounds were easily accessed, eliminating the need for complex processes and patterning techniques to produce superhydrophobic fluorinated surfaces. The transparent PFPEM-POSS coating exhibited a hierarchical micro-nano scale 
structure as well as good superhydrophobic properties on the treated substrate. Moreover, after 5000 cycles of abrasion testing, the PFPEM-POSS coatings maintained their superhydrophobic characteristic. Therefore, PFPEM-POSS, exhibiting superhydrophobicity and excellent durability, may be useful for various functional applications. In addition, the method reported herein using environmentally-friendly fluorinated monomers to improve current industrial coatings can have a wide application in daily life.

\section{Conflicts of interest}

There are no conflicts to declare.

\section{Acknowledgements}

This work was supported by a Major Project of the Ministry of Science and Technology of China [No. 2017YFB0307700], a Major Project of the Science and Technology Research Coordination from Shaanxi Province [No. 2015KTCL01-14], and the Industrialization Cultivation Project of Shaanxi Provincial Education Department [No. 16JF004].

\section{Notes and references}

1 Z. Guo, W. Liu and B. Su, J. Colloid Interface Sci., 2011, 353, 335-355, DOI: 10.1016/j.jcis.2010.08.047.

2 Y. Zhang, Y. Chen, L. Shi, J. Li and Z. Guo, J. Mater. Chem., 2012, 22, 799-815, DOI: 10.1039/C1JM14327A.

3 B. Wang, Y. Zhang, L. Shi, J. Li and Z. Guo, J. Mater. Chem., 2012, 22, 20112-20127, DOI: 10.1039/c2jm32780e.

4 H. Choi, K. C. Park, H. Lee, T. Crouzier, M. F. Rubner, R. E. Cohen, G. Barbastathis and G. H. Mckinley, ACS Appl. Mater. Interfaces, 2017, 9, 8354-8360, DOI: 10.1021/ acsami.6b14631.

5 C. Dorrer and J. Ruhe, Soft Matter, 2009, 5, 51-61, DOI: 10.1039/b811945g.

6 P. Critchlow, R. Litchfield, I. Sutherland, D. Grandy and S. Wilson, Int. J. Adhes. Adhes., 2006, 26, 577-599, DOI: 10.1016/j.ijadhadh.2005.09.003.

7 D. Schaeffer, G. Polizos, D. Smith, D. Lee, S. Hunter and P. Datskos, Nanotechnology, 2015, 26, 055602, DOI: 10.1088/0957-4484/26/5/055602.

8 V. G. Parale, D. B. Mahadik and S. A. Mahadik, Ceram. Int., 2013, 39, 835-840, DOI: 10.1016/j.ceramint.2012.05.079.

9 Y. Chen, Y. B. Zhang, L. Shi, J. Li, Y. Xin, T. T. Yang and Z. G. Guo, Appl. Phys. Lett., 2012, 101, 033701-033704, DOI: 10.1063/1.4737167.

10 T. Verho, C. Bower, P. Andrew, S. Franssila, O. Ikkala and R. H. Ras, Adv. Mater., 2011, 23, 673-678, DOI: 10.1002/ adma.201003129.

11 T. Yanagisawa, A. Nakajima, M. Sakai, Y. Kameshima and K. Okada, Mater. Sci. Eng., B, 2009, 161, 36-39, DOI: 10.1016/j.mseb.2008.11.016.

12 X. Deng, L. Mammen, Y. F. Zhao, P. Lellig, K. Mullen, C. Li, H. J. Butt and D. Vollmer, Adv. Mater., 2011, 23, 2962, DOI: 10.1002/adma.201100410.
13 M. Long, S. Peng, W. Deng, X. Yang, K. Miao, N. Wen, X. Miao and W. Deng, J. Colloid Interface Sci., 2017, 508, 18-27, DOI: 10.1016/j.jcis.2017.08.027.

14 Y. C. Jung and B. Bhushan, ACS Nano, 2009, 3, 4155-4163, DOI: $10.1021 / \mathrm{nn} 901509$ r.

15 J. Chen, M. Long, S. Peng, X. Yang and W. Deng, New J. Chem., 2017, 41, 1334-1345, DOI: 10.1039/c6nj03696a.

16 M. Long, S. Peng, J. Chen, X. Yang and W. Deng, Colloids Surf., A, 2016, 507, 7-17, DOI: 10.1016/ j.colsurfa.2016.07.085.

17 G. Li, L. Wang, N. Hanli and C. U. Pittman, J. Inorg. Organomet. Polym., 2001, 11, 123, DOI: 10.1023/ A:1015287910502.

18 J. M. Mabery, A. Vij, S. T. Iacono and B. D. Viers, Angew. Chem., Int. Ed., 2008, 47, 4137-4140, DOI: 10.1002/ anie. 200705355 .

19 S. T. Iacono, S. M. Budy, D. W. Smith and J. M. Mabry, J. Mater. Chem., 2010, 20, 2979-2984, DOI: 10.1039/B924337B.

20 H. Wang, Y. Xue, J. Ding, L. Feng, X. Wang and T. Lin, Angew. Chem., Int. Ed., 2011, 50, 11433-11436, DOI: 10.1002/ anie.201105069.

21 J. Scheirs, Modern Fluoropolymers, John Wiley \& Sons, Ltd., New York, 1997.

22 V. A. Ganesh, A. S. Nair, H. K. Raut, T. Tan, C. He, S. Ramakrishna and J. Xu, J. Mater. Chem., 2012, 22, 18479-18485, DOI: 10.1039/c2jm33088a.

23 E. Sahlin, A. T. Beisler, S. T. Woltman and S. G. Weber, Anal. Chem., 2002, 74, 4566-4569, DOI: 10.1021/ac025622c.

24 B. Ameduri, B. Boutevina and G. Kostov, Prog. Polym. Sci., 2001, 26, 105-187, DOI: 10.1016/S0079-6700(00)00044-7.

25 J. C. Yarbrough, J. P. Rolland, J. M. DeSimone, M. E. Callow, J. A. Finlay and J. A. Callow, Macromolecules, 2006, 39, 25212528, DOI: $10.1021 / \mathrm{ma} 0524777$.

26 M. Skrzypiec, A. Wamke, K. Dopierała and K. Prochaska, Colloids Surf., A, 2018, 556, 140-147, DOI: 10.1016/ j.colsurfa.08.033.

27 Y. Li, Z. Zhang, X. Zhu, X. Men, B. Ge and X. Zhou, Appl. Surf. Sci., 2015, 328, 475-481, DOI: 10.1016/j.apsusc.2014.12.086.

28 A. Maurizio, E. E. Maria and I. Barbara, Macromol. Chem. Phys., 1998, 199, 91901-91907, DOI: 10.1002/(SICI)15213935(19980901)199:9<1901::AID-MACP1901>3.0.CO.

29 J. T. Garrett, J. Runt and J. S. Lin, Macromolecules, 2000, 33, 6353-6359, DOI: 10.1021/ma000600i.

30 L. Yao, T. Yang and S. Y. Cheng, J. Appl. Polym. Sci., 2010, 115, 3500-3507, DOI: 10.1002/app.30526.

31 S. Xiong, X. Guo, L. Li, S. Wu, P. Chu and Z. Xu, J. Fluorine Chem., 2010, 131, 417-425, DOI: 10.1016/ j.jfluchem.2009.12.015.

32 A. Xue, X. Guo, J. Ma and S. Jia, ACS Appl. Mater. Interfaces, 2015, 7, 8251-8259, DOI: 10.1021/acsami.5b01426.

33 C. Aulin, S. H. Yun, L. Wagberg and T. Lindstrom, ACS Appl. Mater. Interfaces, 2009, 1, 2443-2452, DOI: 10.1021/ am900394y.

34 S. Block, P. Hupfield, Y. Itami, E. Kitaura, D. Kleyer, T. Masutani and Y. Nakai, Dow Corning, Form No. 26-162501, http://www.dowcorning.com. 